\section{Undifferentiated Cardiac Sarcoma on the Mitral Valve Mimicking Myxoma}

Pengfei Yue; Zhian Chen;

Ziqian Xu, MD; Yucheng Chen, MD

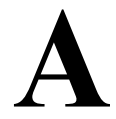

53-year-old male presented with progressive fatigue and dyspnea for 2 months. On admission, echocardiography revealed a leaf-shape mass anchored to the mitral valve (Figure A), which was causing moderate mitral stenosis with an effective orifice area of $1.5 \mathrm{~cm}^{2}$ and forward blood flow acceleration with $V_{\max }=2.3 \mathrm{~m} / \mathrm{s}$ (Figure B). Cardiac magnetic resonance confirmed the mass and found that it moved with the opening and closing of the mitral valve (Figure C). The mass measured as $3.1 \times 2.4 \mathrm{~cm}$ and showed intermediate $\mathrm{T} 1$ signal, high $\mathrm{T} 2$ signal, and heterogeneous late gadolinium enhancement (Figure D). Myxoma, a benign tumor, was initially suspected. However, single-photon emission computed tomography (PET-CT) scan demonstrated increased glucose metabolism of the mass and strongly suggested malignancy (Figure E). Therefore, the patient underwent concurrent mass resection and mitral valve replacement. Hematoxylin-eosin staining revealed obvious atypia and mitotic figures, a mixture of round, fat spindle, and pleomorphic tumor cells (Figure F). Immunohistochemistry staining was positive SMA (Figure G) and CDK (Figure H), whereas CD34, S-100, and MDM2 yielded negative results. This suggested the diagnosis of primary cardiac undifferentiated sarcoma. Chemotherapy was initiated and cranial radiotherapy was added 1 year later due to metastasis to the right temporal lobe (Figure I).

Undifferentiated sarcoma originating from the mitral valve is extremely rare. ${ }^{1}$ As in the present report, a mass without obvious signs of invasiveness or pericardial effusion may resemble a benign tumor. Thus, PET-CT scan and biopsy further help to identify the nature of masses on the mitral valve.

\section{Acknowledgments}

This work was supported by the 1.3.5 Project for Disciplines of Excellence, West China Hospital [grant no. ZYJC18013]; the National Natural Science Foundation of China [82000353], and the China Postdoctoral Science Foundation (2019M663523).

\section{Disclosures}

All authors report no conflicts of interest or disclosures.

\section{Ethics Committee}

West China Hospital [2016(335)]

\section{Reference}

1. Taguchi S. Comprehensive review of the epidemiology and treatments for malignant adult cardiac tumors. Gen Thorac Cardiovasc Surg 2018; 66: 257-262.

Received August 4, 2021; revised manuscript received August 7, 2021; accepted August 10, 2021; J-STAGE Advance Publication released online September 28, 2021 Time for primary review: 1 day

Department of Biotherapy, Cancer Center, State Key Laboratory of Biotherapy (P.Y., Z.C.), Department of Radiology (Z.X.), Department of Cardiology (Y.C.), West China Hospital, Sichuan University, Chengdu, Sichuan, China

The first two authors contributed equally to this work (P.Y., Z.C.).

Mailing address: Yucheng Chen, MD, FACC, Professor, Department of Cardiology, West China Hospital, Sichuan University, No.37, Guo Xue Xiang, Chengdu, Sichuan, 610041, P. R. China. E-mail: chenyucheng2003@163.com

All rights are reserved to the Japanese Circulation Society. For permissions, please e-mail: cj@j-circ.or.jp ISSN-1346-9843

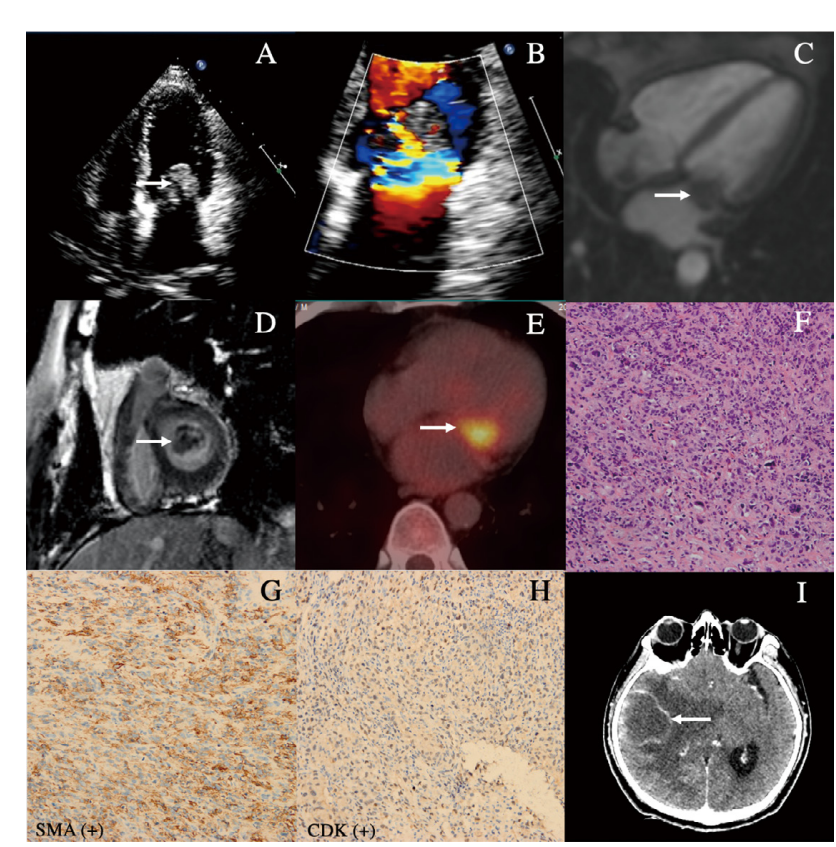

Figure. Transthoracic echocardiogram showing a leafshaped mass anchored to the mitral valve (arrow, A). Color Doppler imaging shows moderate mitral stenosis with an effective orifice area of $1.5 \mathrm{~cm}^{2}$ and forward blood flow acceleration with a $V_{\max }$ of $2.3 \mathrm{~m} / \mathrm{s}(\mathbf{B})$. Cardiac magnetic resonance imaging shows the mass with heterogeneous late gadolinium enhancement (C,D, arrows) and PET-CT scan show increased glucose metabolism of the mass (E). Hematoxylin and eosin staining (×200) shows obvious atypia and mitotic figures and a mixture of round, fat spindle, and pleomorphic tumor cells (F). Immunohistochemical staining (×200) positive for SMA and CDK $(\mathbf{G}, \mathbf{H})$. Right temporal lobe metastasis detected 1 year after cardiac surgery (I). 